\title{
All-fiber transform-limited spectral compression by self- phase modulation of amplitude-shaped pulses
}

\author{
Julien Fatome ${ }^{1}$, Bertrand Kibler ${ }^{1}$, Esben R. Andresen ${ }^{2}$, Hervé Rigneault ${ }^{2}$ and Christophe Finot ${ }^{1}{ }^{*}$ \\ ${ }^{I}$ Laboratoire interdisciplinaire Carnot de Bourgogne, UMR 6303 CNRS - Université de Bourgogne, Dijon, France \\ ${ }^{2}$ Institut Fresnel, UMR 6133 CNRS, Aix-Marseille Université, Domaine Universitaire de St Jérôme 13397 Marseille Cedex 20, France \\ "christophe.finot@u-bourgogne.fr
}

\begin{abstract}
We demonstrate efficient spectral compression of picosecond pulses in an all-fiber configuration at telecommunication wavelengths. Thanks to parabolic pulse shaping, a spectral compression by a factor 12 is achieved with an enhanced Strehl ratio.

OCIS codes: 190.4370 (nonlinear optics, fibers), 320.5540 (pulse shaping), 070.4340 (optical signal processing)
\end{abstract}

\section{Introduction}

Due to the strong light confinement over a large distance, optical fibers are an ideal medium to observe and take advantage of the self phase modulation (SPM) induced by Kerr nonlinearity [1]. Highly coherent supercontinuum and ultrashort pulses have therefore benefited from the large spectral expansion experienced in highly nonlinear fibers. A less-known potential application of SPM relies on the configuration where the initial pulse has a negative linear chirp corresponding to an anomalous dispersive medium. In such a configuration, the SPM-induced chirp cancels the initial frequency modulation so that long and short wavelengths redistribute toward the center wavelength, leading to a spectral narrowing of the pulse [2,3]. The performances of the spectral compression process crucially depends on the initial pulse shape and routinely used hyperbolic secant or Gaussian temporal intensity profiles lead to the presence of large spectral pedestals. To overcome this issue, the optimum pulse shape has been theoretically found to be a parabola, which has recently been experimentally validated by using femtosecond pulses evolving in a passive photonic crystal fiber around $1 \mu \mathrm{m}$ [4]. In this contribution, we provide a new illustration of the benefits brought by an initial shaping of the temporal intensity profile. Our experiments explore a completely different range of input parameters (initial wavelength, duration and peak power as well as typical fiber lengths) and a high-quality spectral compression by a factor above 10 is demonstrated in a passive fiber.

\section{Experimental set-up}

The experimental setup is sketched in Fig. 1a. A 2.4 ps pulse train is delivered by a mode-locked fiber laser (MLL) running at a repetition rate of $10 \mathrm{GHz}$ at a central wavelength of $1552.5 \mathrm{~nm}$. The pulses have a transform-limited hyperbolic secant profile. The repetition rate is then decreased down to $1.25 \mathrm{GHz}$ by means of an intensity modulator (IM) and a phase modulator (PM) is used to avoid Brillouin backscattering. The shaping of the amplitude spectral profile is achieved by a liquid crystal on silicon device ((Finisar WaveShaper) and the anomalous linear chirp is imprinted by a 3900-m segment of standard single mode fiber (SMF). The initial pulses are thus stretched to a temporal duration of $70 \mathrm{ps}$ and are characterized in intensity by a fast oscilloscope (see Fig. 1b) before being amplified up to $30 \mathrm{dBm}$ of average power by a high-power erbium doped fiber amplifier (EDFA). The output power is varied by an optical variable attenuator (OVA) controlled by a computer (PC) and synchronized to the optical spectrum analyzer (OSA). Finally, the spectral narrowing takes place in a 1-km long highly nonlinear fiber (HNLF) with an attenuation of $1 \mathrm{~dB} / \mathrm{km}$, a nonlinear coefficient of $10 / \mathrm{W} / \mathrm{km}$ and a normal dispersion.
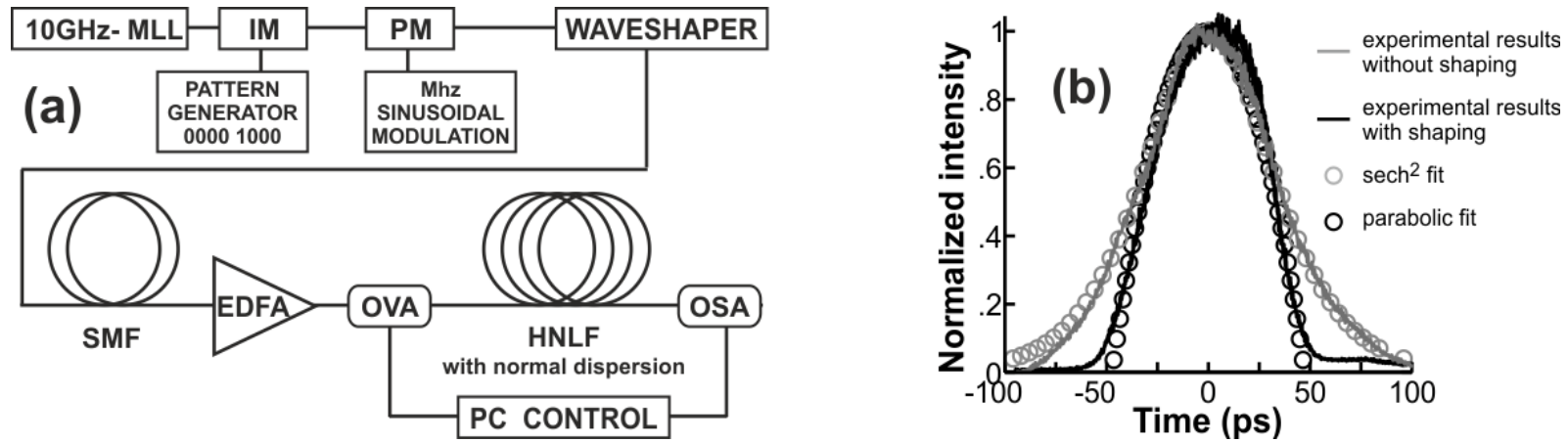

Fig. 1: (a) Experimental setup. (b) Intensity profiles after the SMF with and without shaping compared with sech ${ }^{2}$ and parabolic fits. 


\section{Experimental results}

Figures $2 \mathrm{ab}$ describe the evolution of the output spectral profiles as a function of the input pulse peak-power for the two pulse shapes under investigation. These figures outline the significant influence of the initial shape on the compression behavior. If the spectral width and the power required for optimum compression are comparable, the presence of sidelobes and oscillations is clearly accentuated in the case of a $\operatorname{sech}^{2}$ profile. One can also make out that the spectral brightness is enhanced using a parabolic input pulse. Moreover, the spectral expansion following the point of optimum compression differs significantly.

Details of the output spectrum at the optimum compression point (Fig. 2c) show that compared to input pulses, the power density has then been increased by a factor 9 for parabolic pulses, which represents a twofold improvement of the spectral brightness compared to the $\operatorname{sech}^{2}$ pulses. In both cases, a compression factor ratio of 12.2 is measured and we have checked that the temporal intensity profiles of the output pulses were not affected by the compression process. Therefore, the resulting time-bandwidth product (TBP) is found to be 0.745 . We are thus only $12 \%$ higher than the bandwidth limited case (theoretical TBP of a parabolic pulse is 0.66 ). On the other side, for hyperbolic secant pulses, the resulting TBP is more than twofold above the transform limit condition $(0.315)$.

Figure $2 \mathrm{~d}$ describes more quantitatively the differences between the two initial pulse shapes: we have plotted as a function of the input peak-power the evolution of Strehl ratio defined as the ratio between the actual spectral peak power and the spectral peak power obtained assuming a flat temporal phase of the pulse. The Strehl ratio demonstrates once again the superiority of the parabolic-shaped pulses with a Strehl ratio three times higher than in the sech pulse case. Finally, we show that the root-mean-square (rms) spectral width measured for the parabolic pulse shape as a function of input peak power (Fig 2e) follows a simple but non-trivial parabolic law, confirming an analogy that can be drawn between the spectral compression of a parabolic pulse and the impact of second order dispersion in the temporal domain.
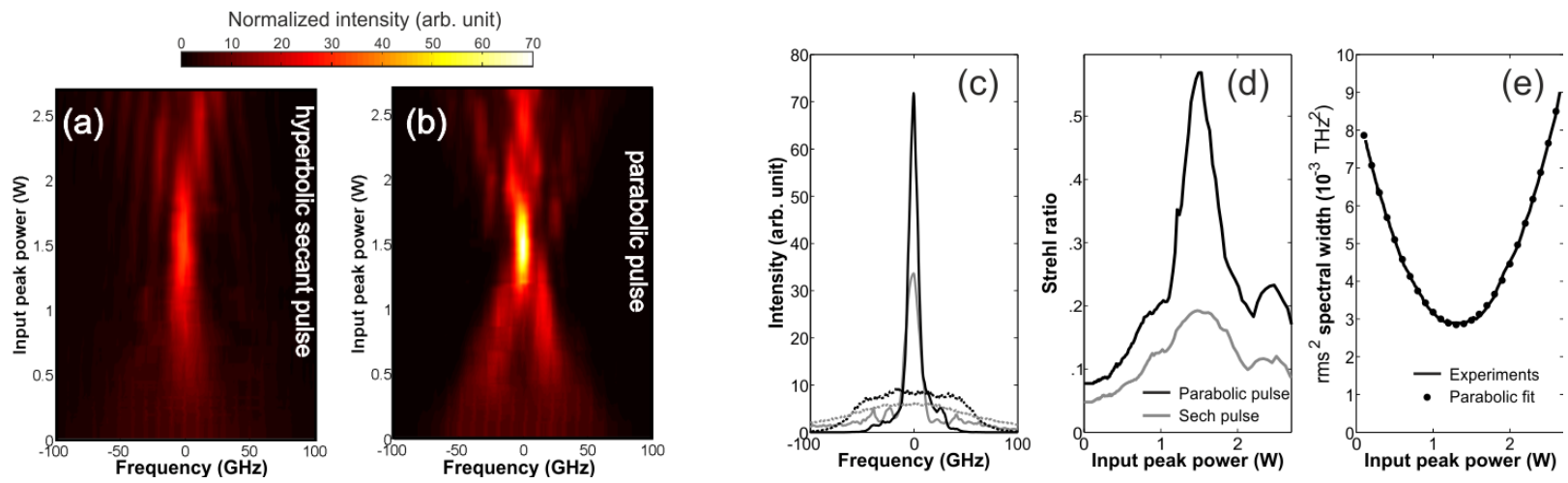

Fig. 2 : (a-b) Experimental evolution of the spectral profile as a function of the input peak power for initial sech ${ }^{2}$ and parabolic profiles. Spectra are normalized so that the energy of the spectra equals 1. (c) Optical spectra obtained at the optimum compression point (solid line) for a sech pulse (grey line) and parabolic shaped pulse (black line) and compared to the input spectra (dashed line). (d) Evolution of the Strehl ratio according to the initial peak-power. (e) Evolution of the rms spectral width according to the input peak power for a parabolic pulse.

\section{Conclusion}

We have demonstrated the spectral compression of picosecond pulses in an all-fiber configuration at telecommunication wavelengths. Using widely available standard single mode fiber as a stretcher to impose the initial linear chirp, a spectral narrowing by a factor above 12 has been achieved for output pulses close to the Fourier transform limit. This compression has been based on a kilometer long highly nonlinear fiber with W-level peak powers and has involved pulse energies well below the nJ level, which is compatible with GHz operation. An initial parabolic pulse shaping has also been shown to significantly improve the results. The quality of the compressed spectra has indeed been enhanced with much lower substructures and an improved Strehl ratio.

\section{References}

[1] G. P. Agrawal, Nonlinear Fiber Optics, Fourth Edition (Academic Press, San Francisco, CA, 2006).

[2] S. A. Planas, N. L. Pires Mansur, C. H. Brito Cruz, and H. L. Fragnito, "Spectral narrowing in the propagation of chirped pulses in singlemode fibers," Opt. Lett. 18, 699-701 (1993).

[3] M. Oberthaler, and R. A. Höpfel, "Spectral narrowing of ultrashort laser pulses by self-phase modulation in optical fibers," Appl. Phys. Lett. 63, 1017-1019 (1993).

[4] E. R. Andresen, J. M. Dudley, C. Finot, D. Oron, and H. Rigneault, "Transform-limited spectral compression by self-phase modulation of amplitude shaped pulses with negative chirp," Opt. Lett. 36, 707-709 (2011). 